\title{
Implicancias de la neuroeducación y desempeño docente: desde la perspectiva del estudiantado
}

\author{
Implications of neuroeducation and teacher performance: from a student perspective
}

Implicações da neuroeducação e do desempenho do professor: da perspectiva do aluno

Humberto Mamani-Coaquira

hmamanic@unap.edu.pe

https://orcid.org/0000-0002-0238-2271

Universidad Nacional del Altiplano, Perú

Wido Willam Condori Castillo

widocondori@unap.edu.pe

https://orcid.org/0000-0001-6569-9148

Universidad Nacional del Altiplano, Perú
Fredy Sosa Gutierrez

fredysosa@unap.edu.pe

https://orcid.org/0000-0001-6473-3877

Universidad Nacional del Altiplano, Perú

Ruth Mery Cruz Huisa

rcruzh@unap.edu.pe

https://orcid.org/0000-0001-7781-0341

Universidad Nacional del Altiplano, Perú

Artículo recibido en junio 2021, revisado en julio 2021, arbitrado en agosto 2021 y publicado en septiembre 2021

\section{RESUMEN}

La investigación relacionó las variables de neuroeducación y desempeño docente, considerando que la formación profesional de los futuros docentes pretende dar a conocer el nuevo modelo educativo, por lo que la enseñanza tradicional ya no tendrá cabida en la universidad actual. El objetivo del estudio fue determinar la relación entre la neuroeducación y el desempeño docente desde la perspectiva de los estudiantes en la escuela profesional de Educación Primaria de la Universidad Nacional del Altiplano Puno (UNAP) durante el 2020. Metodológicamente, la investigación fue de enfoque cuantitativo, de tipo no experimental, el diseño de investigación transeccionales correccionales-causales. Los resultados se sustentaron en la prueba estadística de correlación de Rho de Spearman que es igual a 0,477 , las mismas que trabajaron la prueba de hipótesis. En conclusión, la investigación evidencia que existe una correlación positiva moderada entre la neuroeducación y desempeño de la práctica docente desde la mirada de los estudiantes.

Palabras clave: Cerebro lógico; Desempeño docente; Ejecución; Evaluación; planificación

\section{ABSTRACT}

The research related the variables of neuroeducation and teaching performance, considering that the professional training of future teachers aims to introduce the new educational model, therefore, traditional teaching will no longer have a place in today's university. The objective was to determine the relationship between neuroeducation and teaching performance from the perspective of students in the professional school of Primary Education at the Universidad Nacional del Altiplano Puno (UNAP) during 2020. Methodologically, the research was of quantitative approach, non-experimental type, transectionalcorrectional-causal research design. The results were sustained by the statistical test of Spearman's Rho correlation which is equal to 0.477 , the same that worked the hypothesis test. In conclusion, the research shows that there is a moderate positive correlation between neuroeducation and teaching practice performance from the students' point of view.

Key words: Logical brain; Teaching performance; Execution; Evaluation; planning

\section{RESUMO}

A pesquisa relacionou as variáveis de neuroeducação e desempenho do ensino, considerando que a formação profissional dos futuros professores visa introduzir o novo modelo educacional, de modo que o ensino tradicional não terá mais lugar na universidade de hoje. O objetivo isso foi determinar a relação entre a neuroeducação e o desempenho docente a partir da perspectiva dos estudantes da escola profissional de Ensino Fundamental da Universidade Nacional do Altiplano Puno (UNAP) durante o ano de 2020. Metodologicamente, a pesquisa é de abordagem quantitativa, não-experimental, transeccional-correcional-causal. Os resultados foram sustentados apoiados pelo teste estatístico da correlação Rho da Spearman, que é igual a 0,477 , o mesmo que funcionou no teste de hipóteses. Em conclusão, a pesquisa mostra que existe uma correlação positiva moderada entre o desempenho da neuroeducação e a prática do ensino do ponto de vista dos estudantes.

Palavras-chave: Cérebro lógico; desempenho do ensino; implementação; avaliação; planejamento 


\section{INTRODUCCIÓN}

El estudio se centró en la vinculación que existe entre la neuroeducación y el desempeño docente desde la mirada de los actores directos, que son los estudiantes. En la actualidad, la neuroeducación ha cobrado suficiente notoriedad y está implicada a todas las actividades que desarrolla el hombre. Dada la complejidad en la educación, los investigadores la abordan desde distintasaristas.ParaGonzáles(2016), es un modelo educacional basado en el cerebro al cual apostamos por cuanto solo se aprende con este órgano. A través de la neurociencia cognitiva, la psicología cognitiva y la didáctica educativa (Plos, 2019). En esta línea, Pallarés-Domínguez (2016) menciona que ayuda a los docentes a conocer el funcionamiento del cerebro y colabora a mejorar el rendimiento cerebral consiguiendo un aprendizaje significativo (Ruiz, 2018). De otro lado, Codina (2014) la neuroeducación debe permitir una educación ética y cívica requerida por las sociedades multiculturales. Una educación centrada en las virtudes procedimentales o universales, que apunta hacia las popularizaciones y apropiaciones indebidas de la neurociencia (Vos, 2016).

En la perspectiva del cerebro triuno, el neurocientífico no debe ignorar al educador, del mismo modo que el educador no debe ignorar los descubrimientos del neurocientífico. Para González (2017), el cerebro triuno es la visión de un cerebro dinámico y global potencia el bienestar y facilita las competencias intrapersonales e interpersonales, ampliando los horizontes en el sistema educativo. todas las inteligencias pueden interactuar en forma integrada y una depende de la otra (Seijo y Barrios, 2012). A partir de esas concepciones se ha podido explicar el comportamiento del ser humano desde una perspectiva más integrada, donde el pensar, sentir y actuar conforman un todo que influye en el desempeño de la persona, tanto en lo personal, laboral, profesional y social. A esto se conoce como la teoría del cerebro total, porque toma en cuenta los dos hemisferios con sus respectivas especializaciones, también el cerebro como el órgano dividido en partes, e integrado en un todo al mismo tiempo (Sindeev, 2018). Es posible responder a los retos que imponen las necesidades de un auténtico aprendizaje innovador y transformador, siempre y cuando se potencien las amplias posibilidades del cerebro humano (Velásquez et al., 2005), por lo tanto, hay que formar grupos e individuos que supongan en sí mismos un gran cerebro total (Estrada et al., 2014). De tal modo que los futuros profesionales acuñen actitudes cognitivas, afectivas y conductuales favorables a la diversidad y respondan a la pluralidad existente (Sosa, 2016).

La neuroeducación asume distintas áreas de estudio, para efectos de la investigación se centrarán en tres dimensiones que son los cerebros; lógico analítico, intuitivos emocional y motoras operacionales. Respecto al cerebro lógico analítico Gregori (2019) refiere que el cerebro izquierdo - neocortical - frontal como función lógico-racional, analítica, crítica, semiconsciente o potencial para ser consciente y, por tanto, más responsable. Es la porción de evolución histórica más reciente y más costosa, y de consistencia más frágil o inestable. Solamente esta porción cerebral es verbal, intencional y maneja mejor lo simbólico - teórico o lo abstracto. Portellano (2009) afirma que en términos generales el hemisferio izquierdo es dominante para el lenguaje. De otro lado, mide la capacidad intelectual de las personas a través de pruebas que sólo valoran el desarrollo de la mente en dos áreas del conocimiento como son la verbal y la matemática. (Arias et al., 1999). 
Una manera de evidenciar el cerebro lógico analítico, es cuando se participan en conferencias, congresos, simposios, debates y eventos similares en el que los académicos participan. Estos acontecimientos académicos imprimen textos orales, en el que se producen una estructura y unas características léxico-gramaticales particulares, en el que la comunicación con la audiencia es inmediata. De otra parte, se usa los números, estadística, porcentajes (inteligencia lógica matemático). Por su parte, Armstrong (2013) menciona que el uso de los números con eficacia y de razonar bien, conlleva a desarrollar inteligencias que incluye la sensibilidad a patrones y relaciones lógicas, afirmaciones y proposiciones, funciones y otras abstracciones relacionadas. Los procesos empleados en la inteligencia lógico-matemática incluyen: categorización, clasificación, deducción, generalización, cálculo y prueba de hipótesis. Por último, se puede mencionar que contribuyen a lograr una comprensión más adecuada de la lectura los estudiantes del altiplano peruano (HuancaArohuanca et al., 2021).

Con respecto al cerebro intuitivo emocional, Gregori (2019) plantea que el cerebro derecho, místico también va mal porque está perdido en exhibicionismo, en supersticiones, en milagredismo o en populismo metafísico de ondas de nivel alfa o esotérico - mágico. Para Romero (1996), el hemisferio derecho es el que hay que cuidar especialmente si de lo que se trata es de realizar alguna tarea que tenga que ver con el ámbito del arte y en general con la creatividad. Este lado parietal del cerebro, con función racional, emocional, artística, intuitivo y mística. Constituye la porción creativa, imaginativa, el lado sensible o el conjunto privilegiado del artista, del esotérico y del espiritualista. Su modo de percibir y expresarse es por imágenes, metáforas, mitos, y por lenguaje no verbal como en el arte y en los sueños. Además, Barroso y Nieto (1996) la integridad del HD es necesaria para el procesamiento léxico-semántico y para una adecuada relación lenguaje-contexto.

Por su parte Sánchez-López et al. (2015) menciona que las escalas de inteligencia emocional y bienestar psicológico están fuertemente correlacionadas, especialmente las dimensiones de optimismo, logro y autoestima de la inteligencia emocional con la dimensión de bienestar psicológico subjetivo del bienestar psicológico; sin embargo, un hallazgo interesante fue encontrar que el mayor rendimiento académico no parece estar necesariamente asociado a mayor inteligencia emocional. Al mismo tiempo, Mérida-López y Extremera (2020) expresa que los profesores noveles con bajo compromiso ocupacional y bajos niveles de inteligencia emocional informan de una mayor intención de abandono de la enseñanza que sus compañeros con mayores niveles de inteligencia emocional.

No obstante, los estudiantes con mayor empleo de las estrategias productivas de afrontamiento (solución activa, búsqueda de información y guía, $\mathrm{y}$ actitud positiva) tienen mayor puntuación en las variables autoconcepto académico, habilidades sociales e inteligencia emocional (Morales, 2017). Es necesario dejar en claro que el apoyo social y la inteligencia emocional están significativamente relacionados en ambos sexos (Azpiazu et al., 2015).

También a esta problemática se suma el aspecto de la afectividad, pues es una emoción fundamental en los actos de conocer, pensar, actuar y relacionarse (González y González, 2000). El aula de clase es algo más que un espacio de intercambios cognitivos, es un espacio de construcción de actitudes y vivencias subjetivas ocasionadas tanto por el aprendiz y por quien enseña. 
Respecto al cerebro motor operacional, Gregori (2019) plantea que el lado central operativo se ha desgobernado y se ha impuesto como amo y señor violento de la vida y del ecosistema, con sus mitos de desarrollo y riqueza, rompiendo todo y cualquier límite impuesto por la racionalidad y por el ecosistema. Los manipuladores de este poder están produciendo unos pocos ricachones, y no riqueza para todos. Este régimen del cerebro central más fuerte y más violento tiene como respuesta a la violencia de los depredadores que se van indignado. En otro orden de ideas, Torres (2014) plantea que es una red inteligente, que será aquella en la que tomen lugar múltiples interacciones entre empresas y una permanente retroalimentación tanto positiva como negativa. El principal objetivo del docente no es crear un juego, sino que el estudiante conozca en experiencia propia un proceso de innovación y difusión. (Dehesa-De Gyves, 2018).

El cerebro es lo que es la persona o, al revés, cada persona es lo que es su cerebro. Esto quiere decir que la energía que entra al cuerpo humano por la vía de los alimentos, en su mayor proporción es utilizada por el cerebro. Cuando estamos durmiendo, el cerebro disminuye su actividad, pero nunca cesa; sin embargo, el cerebro como cualquiera de nuestros órganos del cuerpo, se desarrolla y madura mientras crecemos; experimenta un desarrollo rápido poco después del nacimiento y a lo largo de la infancia temprana y tiene periodos sensibles para el aprendizaje (Velásquez et al., 2009).

Es preciso acotar que en la lógica del cerebro operacional, Vidarte et al. (2011) afirma que la actividad física es una herramienta eficaz y efectiva en la promoción de la salud. Sin embargo, son manifestaciones culturales presentes en todos los grupos y sociedades, suponiendo una parte importante del bagaje socio-cultural del individuo (Prieto, 2011). Ruiz (2016) resalta la gimnasia cerebral o Brain Gym funciona bajo la idea de que encontrando los movimientos correctos del cuerpo se pueden superar los problemas de aprendizaje. Esto permite un aprendizaje integral, usando todo el cerebro en conjunción con el cuerpo (Ibarria, 2007). Al mismo tiempo Romero et al. (2014) la aplicación efectiva de una estrategia instruccional basada en la gimnasia cerebral aumenta los niveles de creatividad en los estudiantes.

De otro lado, la investigación abordó el desempeño docente, Barrientos (2014) expresa que se trata de un panorama caótico y caotizante de la sociedad en que vivimos, requiere cambiar a una educación basada en la verdad, bondad, justicia y libertad para una sociedad con una filosofía de la sustentabilidad. Sin embargo, la labor de los educadores ocupa un lugar fundamental en la búsqueda de una educación de calidad, situada y pertinente (Dimaté et al., 2017).

La efectivización de la evaluación del desempeño docente en la UNA-Puno, se da según el reglamento de evaluación de desempeño docente, aprobado por Resolución Rectoral No 1243- 2018-R-UNA, que rige a partir del año 2018. La finalidad está establecida en el Art. $2^{\circ}$ el cual es "garantizar la implementación de mecanismos que evalúen el desempeño docente con la finalidad de identificar necesidades de capacitación y perfeccionamiento, de manera progresiva, permanente y sostenida en el marco de una cultura de calidad institucional". En este proceso de evaluación al desempeño docente, están implicados los estudiantes y dicha evaluación está monitoreada por el director de la Escuela Profesional. Los criterios de evaluación del desempeño docente están referidos a los siguientes aspectos de la planificación, ejecución y evaluación del proceso de enseñanza aprendizaje. Que para el estudio son las dimensiones de estudio y análisis. 
Respecto a la planificación Escanero (2019) manifiesta que la planificación viene a ser secuencias didácticas adecuadas que permitan desarrollar los contenidos conceptuales, procedimentales y actitudinales del módulo, materia o tema; compatibilizando ideas en su propia diagramación (Monti, 2020). Cuenca y Quintana (2014) sostienen que es importante realizar una planificación con diversas actividades que, sincronizadas adecuadamente, permitan conseguir las competencias formativas de la asignatura.

Vale la pena aclarar que se incluyó una posible guía docente que cubrió un amplio abanico de competencias con un planteamiento metodológico a partir de un aprendizaje a través de tareas o proyectos (García, 2013). Adicionalmente, Meneses (2019) menciona que están directamente relacionados con el dominio conceptual y disciplinar del docente, por ello debe tener establecidas claramente las competencias (objetivos y actividades) del curso, módulo o tema, así como la secuenciación de las mismas y las razones de ello (Escanero, 2019).

La ejecución del currículo se refiere a la relación con la puesta en marcha de las acciones planificadas, en esencia alude al cumplimiento de las tareas comprometidas, la forma en que se logran los aprendizajes que adquieren los estudiantes, y la satisfacción respecto al proceso y al clima en el cual se realiza la actividad docente. La enseñanza ayuda a comprender las diferentes formas de conocer, conceptuar, sentir y actuar que individualizan el aprendizaje de nuestros estudiantes. Hervás (2006) manifiesta que la calidad de la enseñanza refiere a la importancia de vincular los estilos de aprendizaje y estilos de enseñanza. Alude a la integración de conocimientos, habilidades, motivos y valores que se expresan en un desempeño profesional eficiente, ético y de compromiso social. Esto implica el manejo de estrategias de enseñanza, que es relevante y de vital significado en los procesos cognitivos, a su vez, el logro de los resultados está vinculada a la evaluación (Sosa y Vilca, 2021).

Villada y Serna (2010) mencionan que las estrategias metodológicas novedosas dinamizan los procesos de planeación. De otro lado, motivan el aprendizaje (Míguez, 2010). Así mismo, MorenoPinado y Velázquez (2017) la estrategia didáctica permiten la formación integral en el proceso de enseñanza-aprendizaje. Las cuáles serán más propicios para obtener ciertos resultados en los estudiantes (Albarrán et al., 2018). La estrategia didáctica va mucho más allá de la búsqueda y la obtención de un aprendizaje eficiente (Hernández, 2018). Para, Fernández (2013) cualquier material elaborado con la intención de facilitar los procesos de enseñanza y aprendizaje. A su vez Vargas (2017) refiere al uso y desarrollo de recursos educativos didácticos, coadyuvará a la intención educativa. En este sentido, Sosa (2018) exige del profesor el dominio de contenidos disciplinares actualizados de su área de desempeño y el fortalecimiento de habilidades, capacidades y competencias con el propósito de garantizar una inserción exitosa a la profesión. De otra parte, las Tecnologías de Información y Comunicación ya sea en el uso o desarrollo de recursos informáticos con o sin conexión a Internet. (Vargas, 2017).

La dimensión de la evaluación como un proceso continuo en la experiencia de enseñanza aprendizaje, es útil por su valor para tomar decisiones. En ese sentido, lo que da sentido a la evaluación es la información que devuelve sobre el programa, la institución, la enseñanza o el aprendizaje. Cuenca y Quintana (2014) señalan que la evaluación debería realizarse únicamente de forma continua, aunque somos conscientes del rechazo que esta idea puede suscitar entre los estudiantes (Mejía, et al., 2019); sin embargo, ofrecer una evaluación confiable, precisa, 
objetiva y auténtica, implica un cierto conocimiento puro, evaluado mediante exámenes, es insuficiente (Celis-Aguilar et al., 2018), encontrando como áreas de mejora las dimensiones de metodología y evaluación (Piedra et al., 2018), al contar con una rúbrica respaldada por la experiencia de profesores brinda la posibilidad de ayudar a otros docentes en el proceso de evaluación. Los estudios indican que los estudiantes que participan en procesos de evaluación formativa favorecen su implicación en el proceso de aprendizaje. Por ello la retroalimentación o feedback durante la experiencia es muy importante en el proceso de comunicación y ajuste de resultados.

En definitiva, el propósito de la investigación fue relacionar la neuroeducación y el desempeño docente, valorada desde la perspectiva de los estudiantes de la Escuela Profesional de Educación Primaria de la UNA - Puno 2020. Los objetivos específicos del trabajo son; explicar el uso del cerebro lógico de los docentes; reconocer el uso del cerebro intuitivo de los docentes; identificar el uso del cerebro operacional de los docentes. Al mismo tiempo buscó explicar si los profesores planifican, ejecutan y evalúan sus actividades académicas conforme lo establece el currículo.

\section{MÉTODO}

Se realizó un estudio descriptivo bajo un enfoque cuantitativo, de corte correlacional, relacionado con el "desempeño docente". El universo poblacional estuvo conformado por 396 estudiantes del I ciclo a X ciclo de la Escuela Profesional de Estudios de Educación Primaria de la Universidad Nacional del Altiplano de Puno de la región de Puno, Perú. Además, la muestra intencional con el que se trabajo fue de 137 universitarios entre varones (30) y mujeres (107).
La técnica empleada fue la observación y el instrumento que se utilizó fue el cuestionario en ambas variables (neuroeducación y desempeño docente) que cada uno consta de 20 ítems, los cuales fueron validados por profesionales con una calificación promedio de 38. De acuerdo a la ficha de estimación, esto se interpreta que tiene un alto nivel de validez en ambos instrumentos. De la confiabilidad del instrumento se obtuvieron los resultados de la prueba piloto que integraron 30 alumnos y posterior a ello se aplicó el coeficiente de alfa de Cronbach con un valor de 0,885 en el instrumento de neuroeducación según la Tabla de confiabilidad se encuentra en un nivel muy bueno; sin embargo, en el instrumento de desempeño docente obtuvo un valor de 0,937 , se valora de acuerdo a la Tabla de confiablidad excelente. En conclusión, los instrumentos son altamente confiable. Para ambos instrumentos se utiliza las escalas referidas: nunca (0), alguna vez (1), casi siempre (2) y siempre (3). El análisis estadístico fue mediante coeficiente de correlación de Spearman.

\section{RESULTADOS Y DISCUSIÓN}

\section{Neuroeducación}

En la Tabla 1 se presentan los resultados de las dimensiones relacionadas con el cerebro lógico, intuitivo y motoras correspondientes a la variable neuroeducación. Luego de un total de 137 estudiantes (100\%) investigados del programa de estudios de educación primaria de la Universidad Nacional del Altiplano

Referente a la dimensión del cerebro analítico. Se puede observar que, 57 estudiantes que representan al $41,61 \%$ evidencian una escala de casi siempre; 52 alumnos (37,96\%), una escala de alguna vez; frente a 20 universitarios (14,60\%), evidencian una 
escala siempre; 8 estudiantes $(5,84 \%)$, consideran una escala de nunca. Esos resultados mencionan que la mayoría de los universitarios $(41,61 \%)$, que los profesores de la EPEP que están ligadas directamente con el cerebro izquierdo; es decir, desarrollan argumentos, averiguan las fuentes, emiten juicios, participan en conferencias y dudan de algunos medios de comunicación.

Referente a la dimensión cerebro intuitivo. Se puede observar que 2 estudiantes que representan el 1,46\% evidencian una escala nunca; 26 alumnos (18,98\%), una escala de siempre; 37 universitarios $(27,01 \%)$, consideran una escala alguna vez; frente a los 72 aprendices que evidencian el 52,55\% estiman una escala siempre y siendo el mayor porcentaje, se caracterizan los catedráticos que promueven el arte, la creatividad, con visión futurista, decisiones y propuestas.

Con referente a la dimensión de cerebro operacional. 64 estudiantes que representan al 45,26\% evidencian la escala casi siempre; 40 alumnos (29,20\%), una escala alguna vez; 32 universitarios $(23,36 \%)$, una escala siempre; frente a 3 aprendices (2,19\%) mencionan la escala de nunca. Dichos resultados indican que la mayoría $(45,26 \%)$ se identifican por poseer una buena organización, disciplina, consciencia, son empáticos, dominio tecnológico, acompañamiento y Upaya coaching.

Tabla 1. Resultados de las dimensiones de la neuroeducación.

\begin{tabular}{ccccccc}
\hline & \multicolumn{2}{c}{ Cerebro Lógico - Analítico } & \multicolumn{2}{c}{ Cerebro Intuitivo - } & \multicolumn{2}{c}{ Cerebro Motor - } \\
Escalas & $\mathbf{f}$ & $\mathbf{\%}$ & $\mathbf{f}$ & $\mathbf{\%}$ & $\mathbf{f i}$ & $\mathbf{\%}$ \\
\hline Nunca (0) & 8 & 5,84 & 2 & 1,46 & 3 & 2,19 \\
Alguna vez (1) & 52 & 37,96 & 37 & 27,01 & 40 & 29,20 \\
Casi siempre (2) & 57 & 41,61 & 72 & 52,55 & 62 & 45,26 \\
Siempre (3) & 20 & 14,60 & 26 & 18,98 & 32 & 23,36 \\
Total & $\mathbf{1 3 7}$ & $\mathbf{1 0 0 , 0}$ & $\mathbf{1 3 7}$ & $\mathbf{1 0 0 , 0}$ & $\mathbf{1 3 7}$ & $\mathbf{1 0 0 , 0}$ \\
\hline
\end{tabular}

Fuente: Encuesta realizada a los estudiantes de la Escuela Profesional de Educación Primaria, UNA-2021.

\section{Desempeño docente}

La Tabla 2 se presentan los resultados de las dimensiones relacionadas con planificación, ejecución y evaluación propios de la variable desempeño docente. Luego de un total de 137 estudiantes (100\%) investigados del programa de estudios de educación primaria de la Universidad Nacional del Altiplano.
En la dimensión planificación. Se puede observar que 5 estudiantes que representan el 3,65\% evidencian una escala nunca; 25 alumnos (18,25\%), una escala de alguna vez; 47 universitarios (34,31\%), consideran una escala siempre; frente a los 60 aprendices que evidencian el $43,80 \%$ consideran una escala casi siempre y siendo el mayor porcentaje, se caracterizan que los catedráticos socializan el sílabo 
direccionado de acuerdo a las cartas descriptivas, de igual forma las competencias se direccionan al perfil de egreso, donde se incorpora las sugerencias y tomando en cuenta las bibliografías actualizadas

En la dimensión ejecución. 57 estudiantes que representan al 41,61\% evidencian la escala siempre; 54 alumnos (39,42\%), una escala casi siempre; 25 universitarios $(18,25 \%)$, una escala alguna vez; frente a 1 aprendices $(0,73 \%)$ mencionan la escala de nunca. Dichos resultados indican que la mayoría $(41,61 \%)$, se identifican los maestros en poseer clases de manera activa, motivada y utilizan estrategias de indagación o generar conocimientos previos, estrategias de elaboración de información, organización, desarrollo de competencias, formación en valores y actitudes, recursos educativos y tecnológicos.
En la dimensión evaluación. Se puede observar que, 62 estudiantes que representan al $45,26 \%$ evidencian una escala de casi siempre; 51 alumnos (37,23\%), una escala de siempre; frente a 23 universitarios (16,79\%), evidencian una escala alguna vez; 1 aprendiz $(0,73 \%)$, consideran una escala de nunca. Esos resultados mencionan que la mayoría de los universitarios (45,26\%), que los docentes de la EPEP que se caracterizan directamente con la evaluación; es decir, evaluando de acuerdo a los criterios de desempeño, promoviendo el uso de instrumentos adecuados para evaluar el aprendizaje, manejando las técnicas de análisis y valoración de producto, organizando el conocimiento, portafolio, análisis de contenido, usando la estrategia de retroalimentación y comunicando los resultados del aprendizaje.

Tabla 2. Resultados de las dimensiones de la neuroeducación.

\begin{tabular}{ccccccc}
\hline \multirow{2}{*}{ Escalas } & \multicolumn{2}{c}{ Planificación } & \multicolumn{2}{c}{ Ejecución } & \multicolumn{2}{c}{ Evaluación } \\
& $\mathbf{f i}$ & $\mathbf{6}$ & $\mathbf{f i}$ & $\mathbf{\%}$ & $\mathbf{f i}$ & $\mathbf{\%}$ \\
\hline Nunca (0) & 5 & 3,65 & 1 & 0,73 & 1 & 0,73 \\
Alguna vez (1) & 25 & 18,25 & 25 & 18,25 & 23 & 16,79 \\
Casi siempre (2) & 60 & 43,80 & 54 & 39,42 & 62 & 45,26 \\
Siempre (3) & 47 & 34,31 & 57 & 41,61 & 51 & 37,23 \\
Total & $\mathbf{1 3 7}$ & $\mathbf{1 0 0 , 0}$ & $\mathbf{1 3 7}$ & $\mathbf{1 0 0 , 0}$ & $\mathbf{1 3 7}$ & $\mathbf{1 0 0 , 0}$ \\
\hline
\end{tabular}

Fuente: Encuesta aplicada a los estudiantes de la Escuela Profesional de Educación Primaria, UNA-2021. 


\section{Prueba de hipótesis}

La prueba de correlación de Spearman tiene como requisito fundamental realizar el supuesto de normalidad para datos de la variable cuantitativa. Es por ello, que es necesario la prueba de kolmogorovSmirnov de contraste para la normalidad.

Planteamiento de la Hipótesis:

$\mathbf{H}_{0}$ : No existe correlación entre los puntajes obtenidos de la neuroeducación y desempeño docente en la Escuela Profesional de Educación Primaria de la Universidad Nacional del Altiplano - Puno, 2021.
$\mathbf{H}_{1}$ : Existe correlación entre los puntajes obtenidos de la neuroeducación y desempeño docente en la Escuela Profesional de Educación Primaria de la Universidad Nacional del Altiplano - Puno, 2021.

Establecer un nivel de significancia

$$
\alpha=0,05 \quad=5 \% \text { margen de error. }
$$

Estadístico de prueba y toma de decisión

Se asumió la correlación Spearman. (Ver Tabla 3.)

Tabla 3. Correlación entre la neuroeducación y desempeño docente.

\begin{tabular}{llcc}
\hline & & Nueroeducación & Desempeño Docente \\
\hline \multirow{3}{*}{ Rho de Spearman } & Coeficiente de correlación & 1,000 & $0,477^{* *}$ \\
& Sig. (bilateral) & $\cdot$ & 0,000 \\
& $\mathrm{~N}$ & 137 & 137 \\
\hline
\end{tabular}

**. La correlación es significativa en el nivel 0,01 (bilateral).

\section{Decisión estadística.}

El coficiente de correlación es Rho $=0,477^{* *}$, ese resultado según la escala de valores de correlación de Spearman, indica que la correlación entre las variables de investigación es positiva moderada, aceptando la hipótesis alterna; donde la probabilidad de la significancia es 0.01 , por el cual la neuroeducación utilizadas mejorara el desempeño docente. Puesto que, las variables actúan conjuntamente en los docentes de la EPEP de Puno. En términos más definidos, existe una moderada correlación entre la neuroeducación y el desempeño docente desde la perspectiva en los estudiantes de la Escuela Profesional de Educación Primaria UNAP - 2020.

\section{Discusión}

Los resultados de la investigación muestran la relación de las variables con el coeficiente de correlación de Rho $=0,477$, señala que es positiva moderada. También cabe destacar que los resultados se asemejan a la investigación realizada por Sánchez et al., (2020) donde se evidencia el resultado un valor de $r=0,321$, donde existe una correlación moderada en el predomino cerebral y el rendimiento académico. Así mismo, es importante mencionar los resultados que se parecen a los resultados de Dorregaray (2020) en su estudio demostraron que existe relación muy alta entre la neuroeducación y las estrategias de aprendizajes que se corroboro con la Rho de Spearman $(0,872)$; 
sin embargo, se puede asegurar que si se utilizan de manera adecuada los cerebros se tiende a mejorar los aprendizajes. Al respecto conviene decir que la neuroeducación y el desempeño docente en ambas variables que si tienen relación. No obstante, los docentes universitarios de la EPEP, logran desarrollar adecuadamente el cerebro triuno (cerebro lógico, intuitivo y operacional). Así mismo González (2017) da a deducir que es el enfoque de un cerebro emprendedor e integral donde facilita las competencias Intrapersonal e Interpersonales, aumentando las perspectivas en el sistema universitario frente a su desempeño docente.

En cuanto a la dimensión del cerebro lógico se encuentra en la escala casi siempre, ya que está encarga de impulsar la realidad, el lenguaje, la matemática, entre otros. Mientras que Arias, et al., (1999) menciona que usualmente se calcula la capacidad intelectual de las personas a través de pruebas que sólo valoran el desarrollo de la mente en dos áreas del conocimiento la verbal y la matemática. Sin embargo, los docentes no deben enfocarse a ello, sino incorporar el pensamiento crítico y reflexivo, donde los universitarios mencionan que los docentes participan frecuentemente en congresos, conferencias, simposios y debates para desarrollar competencias. Es importante dejar claro que los catedráticos tienen buenas explicaciones, saben argumentar y a la vez refutar los conocimientos; no obstante, Cervantes-Barraza et al., (2017) afirman que la objeción tiene un dominio convincente sobre las tesis y están íntimamente relacionadas con lo que está sucediendo en el espacio (Slimovich, 2021). Por eso, los docentes asumen en mejorar sus estrategias ya que se deduce que es constante el aprendizaje.

Por ende, los docentes de la EPEP son creativos e innovadores en la formación de los futuros profesionales, como manifiesta Romero (1996) realizar actividades en el ámbito del arte y en general con la creatividad, también es mantener el espíritu innovador en las clases y frecuentemente insertar la creatividad en el aula (Medina, 2018). De igual forma los estudiantes manifestaron que los catedráticos tienen una visión del mañana en otras palabras presentimientos, sueños, metas y objetivos en el ámbito académico, científico, entre otros; por ello, implica ver mucho con la futurología, en busca del método perfecto para realizar predicciones válidas sobre ello (Vergara et al., 2010). Finalmente desarrollan diversas metodologías que buscan teorizar, estandarizar y operacionalizar (Vergara, Maza, y Fontalvo, 2010).

En tanto, la dimensión del cerebro motor su función es operativa, por ello Gregori (2019) menciona que este cerebro comanda y pone a su servicio los otros dos bloques de igual forma es el principal responsable de la motricidad. Sin embargo, el principal objetivo del docente no es crear un juego, sino que el estudiante conozca en experiencia propia un proceso de innovación y difusión (Dehesa-De, 2018). Entonces se puede decir que los catedráticos hacen uso frecuente del cerebro motor, caracterizados por modos de pensar, estilos de vida propios, diferenciada claramente de los modelos de vida individualista (AñascoHuariccallo, Mamani-Jilaja, y Mamani-Coaquira, 2020).

Cabe agregar que, se puede entender que la planificación es como una ruta para lograr el propósito en unasesión, dondese requiere una amplia investigación y bibliográfico en todos los momentos establecidos dentro de una programación; sin embargo, vale la pena aclarar que Escanero (2019) menciona que la planificación son secuencias didácticas adecuadas que permitan desarrollar los contenidos conceptuales, procedimentales y actitudinales del módulo, materia o tema; compatibilizando ideas en su propia diagramación 
(Monti, 2020). Al mismo tiempo, se entiende que todo docente debe de tener su organización para el éxito de las competencias establecidas en el marco curricular. Distinto de Cuenca y Quintana (2014) que es importante realizar una planificación con diversas actividades que estén sincronizadas adecuadamente.

Cabe resaltar que los catedráticos en la ejecución de su planificación, requieren movilizar una serie de actividades de enseñanza aprendizaje teniendo en cuenta su entorno. Al respecto Ángel y Chauvet (2000) mencionan que en ese proceso de enseñanza aprendizaje se sitúan tres elementos interactuantes e indisolubles: entorno, alumno, docente, que se hayan dirigido hacia el problema del mejoramiento de las técnicas o instrumentos y no hacia el mejoramiento del proceso de enseñanza aprendizaje como un todo (Loredo, 1997). Por lo tanto, la enseñanza como una variable cuyo conocimiento ayuda a comprender las diferentes formas de conocer, conceptuar, sentir y actuar que individualizan el aprendizaje de nuestros estudiantes. Como si fuera poco, todo docente tiene un propósito de movilizar sus capacidades del estudiante y lograr la competencia planteada de esa forma podemos corroborar la calidad educativa de los futuros profesionales; mientras que, Hervás (2006) hace referencia a la calidad de la enseñanza resaltando la importancia de vincular los estilos de aprendizaje y estilos de enseñanza (GonzálezPeiteado, 2013).

Ciertamente, los resultados respecto a la evaluación, los catedráticos tienden a utilizar distintas técnicas e instrumentos para desarrollar la evaluación formativa $y$ un constante acompañamiento a los futuros docentes con el fin de lograr el perfil de egreso. Sin embargo, desde la versión de Cuenca y Quintana (2014), la evaluación debería realizarse únicamente de forma continua. Además, se sabe que se puede observar la evolución de sus competencias; en consecuencia el universitario moviliza una variedad de capacidades al desarrollar una prueba o presentar un trabajo de investigación.

En definitiva, los profesores de la Universidad evalúan de acuerdo a los criterios de desempeño, promoviendo el uso de instrumentos adecuados para evaluar el aprendizaje como la prueba escrita, ficha de observación, lista de cotejo, rubricas entre otros; con el propósito de obtener sus competencias desarrolladas. Por lo tanto, Mejía y otros (2019) señalan que la evaluación es confiable, precisa, objetiva y auténtica; desde esta mirada se debe proponer los trabajos o las pruebas que movilicen destrezas, actitudes, etc. Como si fuera poco ellos manejan las técnicas de análisis y valoración del producto, organización del conocimiento, portafolio, análisis de contenido y documentario de ensayo y monografías; en efecto se acepta como idónea para mejorar los procesos de enseñanza aprendizaje (Pasek y Mejía, 2017).

\section{CONCLUSIONES}

Los aspectos estudiados parecen indicar que la problemática expuesta en los acápites precedentes, pone a flote el desarrollo de la neuroeducación como el uso del cerebro lógico, intuitivo y motor. Todos ellos, de manera directa o indirecta, contribuyen a fortalecer el desempeño docente de la escuela profesional de educación primaria de la Universidad Nacional del Altiplano. Así mismo favorecen en el uso del cerebro triuno a partir del desarrollo de las sesiones de aprendizajes en diversos momentos, pues utilizan óptimas explicaciones, argumentos y saben rebatir, innovan, visionan el futuro, tienden a tener soluciones creativas, tienen consciencia de lo que consumen de igual forma de su descanso y de los ejercicios físicos. Lo más importante del estudio fue relacionar el uso de la neuroeducación 
frente al desempeño docente ya que la valoración de los universitarios se demuestra la utilización del cerebro triuno en sus sesiones de aprendizajes.

En definitiva, el uso adecuado de la neuroeducación contribuye al desarrollo de competencias en los estudiantes; así mismo, en los momentos pedagógicos y didácticos propuestos por los docentes, evidenciando la presentación y socializacióndelsilaboacordea lascartas descriptivas dirigiéndose al logro del perfil de egreso; desde esa misma visión desarrollan actividades de enseñanza aprendizaje de manera creativa y dinámica; poniendo en énfasis la evaluación formativa y los procesos de retroalimentación.

\section{REFERENCIAS}

Albarrán Torres, F., Urrutia Martínez, M., Ibarra Peso, J., Miranda Díaz, C., y Meza Vásquez, S. (2018). Maquetas como estrategia didáctica en estudiantes de la salud. Educacion Medica, 21(3), 198-206. https://doi.org/10.1016/j. edumed.2018.08.003

Añasco-Huariccallo, L. A., Mamani-Jilaja, D., y Mamani-Coaquira, H. (2020). Kachkaniraqmi: el pensamiento social andino paritario frente al pensamiento occidental individualista. Revista Revoluciones, 2(2), 59-71. https://doi.org/ https://doi.org/10.35622/j.rr.2020.02.005

Ángel, M., y Chauvet, R. (2000). Enseñanzaaprendizaje ¿proceso o mito? Iztapalapa 48, 247-260

Arias, N., Guzmán, B., y Payán, A. (1999). Coexistencia de inteligencias múltiples en Beethoven. Colombia Medica, 30(3), 138-141

Armstrong, T. (2013). Inteligencias múltiples en el aula. In Paidós Educación. https:// www.primercapitulo.com/pdf/2017/3381 inteligencias-multiples-en-el-aula.pdf

Azpiazu, L., Esnaola, I., y Sarasa, M. (2015). Capacidad predictiva del apoyo social en la inteligencia emocional de adolescentes. European Journal of Education and Psychology, $8(1), \quad 23-29 . \quad$ https://doi.org/10.1016/j. ejeps.2015.10.003
Barrientos, P. (2014). La educación de los educadores en el desempeño docente. Horizonte de La Ciencia, 4(6), 53-57. https://doi.org/10.26490/ uncp.horizonteciencia.2014.6.91

Barroso, J., y Nieto, A. (1996). Asimetría cerebral: hemisferio derecho y lenguaje asimetría cerebral: hemisferio derecho y lenguaje. Psicología Conductual, 4(3), 285-305. https://www.researchgate.net/ publication/266371006\%0AASIMETRÎA

Celis-Aguilar, E., Dehesa-López, E., y MartínezGonzález, A. (2018). El residente como evaluador del desempe no docente en las especialidades médicas. Educacion Medica, 19(4), 217-222. https://doi.org/10.1016/j.edumed.2017.03.021

Cervantes-Barraza, J., Cabañas-Sánchez, G., y Ordoñez-Cuastumal, J. S. (2017). El Poder Persuasivo de la Refutación en Argumentaciones Colectivas. Boletim de Educação Matemática, 31(59), 861-879. https://doi.org/http://dx.doi. org/10.1590/1980-4415v31n59a01

Codina, M. J. (2014). Neuroeducación: reflexiones sobre neurociencia, filosofía y educación. Postconvencionales, (7), 164-181

Cuenca, C., y Quintana, F. (2014). EEES, TIC y planificación docente: un caso práctico en la Escuela de Ingeniería Informática de la ULPGC. Historia y Comunicación Social, 19(1), 539-550. https://doi.org/http://dx.doi.org/10.5209/rev_ HICS.2014.v19.44983

Dehesa-De Gyves, N. (2018). Revista Internacional de Investigación e Innovación Tecnológica. Revista Internacional de Investigación e Innovación Tecnológica, 6(34), 1-13

Dimaté Rodríguez, C., Tapiero Celis, O., Inés González Rodríguez, C., Rodríguez Rodríguez, R., y Adriana Arcila Cossio, M. (2017). La evaluación del desempeño docente 1 Teaching Performance Assessment A avaliação de desempenho docente. Revista Folios, 1(46), 83-94. http://www.redalyc.org/articulo. oa? id=345951474007

Dorregaray, J. (2020). Nueroeducación y estrategias de aprendizaje en estudiantes de la escuela profesional de tecnología médica de la Universidad Peruana los Andes - 2019 (Tesis de Maestria, Universidad Peruana de Ciencias e Informática). http://repositorio.upci.edu.pe/handle/upci/144 
Escanero, J. F. (2019). Primera clase: guía docente. Educacion Medica, 20(1), 42-48. https://doi.org/ https://doi.org/10.1016/j.edumed.2018.12.001

Estrada, M., Monferrer, D., Segarra, M., y Moliner, M. A. (2014). El trabajo cooperativo en base al modelo del cerebro total: una experiencia docente. Redmarka. Revista de Marketing Aplicado, 12(1), 87-112. https://doi. org/10.17979/redma.2014.01.012.4818

Fernández, F. (2013). Summary for Policymakers. In Intergovernmental Panel on Climate Change (Ed.), Climate Change 2013 - The Physical Science Basis (Vol. 369, pp. 1-30). Cambridge: Cambridge University Press. https://doi. org/10.1017/CBO9781107415324.004

García, S. (2013). La materia Cine y publicidad en los estudios sobre Publicidad y Relaciones Públicas. Propuesta de planificación y metodología docente. Historia y Comunicacion Social, 18(1), 119-131. https://doi.org/http:// dx.doi.org/10.5209/rev_HICS.2013.v18.43953

Gonzáles, C. (2016). Neuroeducación y lingüística: una propuesta de aplicación a la enseñanza de la lengua materna (Tesis Doctoral, Universidad Complutense de Madrid). https://eprints.ucm. es/id/eprint/35929/1/T36890.pdf

González-Peiteado, M. (2013). Los estilos de enseñanza y aprendizaje como soporte de la actividad docente. Revista Estilos de Aprendizaje, $6,51-70$

González, A., y González, M. C. (2000). La afectividad en el aula de clase. Colombia Médica, 31(1), 55-57. http://www.redalyc.org/articulo. oa? id $=28331111$

González, Y. (2017). Teoría del cerebro triuno e inteligencia emocional: Análisis Exploratorio de la Intervención con niños. Educational Psychology Journal, 2(2), 65-72. lib.unnes. ac.id/6871/1/8479.pdf\%0Ahttp://www.albayan. ae

Gregori, W. (2019). Neuroeducación para el éxito (primera ed). Puno: Universidad Nacional del Altiplano-Puno

Hernández, R. M. (2018). La estrategia didáctica frente a los estilos de aprendizaje en la educación superior. Educacion Medica, 19, 227. https://doi. org/10.1016/j.edumed.2017.10.034
Hervás Avilés, R. M. (2006). escenarios educativos. Educatio Siglo XXI, 24(1), 211-216

Huanca-Arohuanca, J. W., Asqui, M. L., Mamani, D., Mamani-Coaquira, H., Huayanca, P. C., y Charaja, F. (2021). Habilidades lingüísticas y comprensión lectora en la oquedad del siglo XXI: una mirada a la Institución Educativa Politécnica de Puno - Perú. Horizontes, 5(18), 537-555. https://doi.org/Https://doi. org/10.33996/revistahorizontes.v5i18.194

Ibarria, L. M. (2007). Aprende mejor gimnasia cerebral (11th ed.)

Loredo, L. P. (1997). La evaluación dentro del proceso enseñanza-aprendizaje

Medina, A. (2018). Creatividad: estrategias y técnicas creativas empleadas en educación universitaria. Revista de Investigación, 42(94), 34-54. https://www.redalyc.org/ journal/3761/376160142002/376160142002.pdf Mejía, G. P., López, M. V., Hernandez-Rangel, E., y Cerano, J. L. (2019). Diseño de un modelo de evaluación mediante la integración de tecnología inmersiva y a distancia. Educacion Medica, 20(3), 140-145. https://doi.org/https:// doi.org/10.1016/j.edumed.2018.02.009

Meneses, M. C. (2019). From historic knowledge to school knowledge. Teacher planning for the teaching of history and social sciences. Historia $y$ Memoria, 1(18), 289-313. https://doi.org/https:/ doi.org/10.19053/20275137.n18.2019.7447

Mérida-López, S., y Extremera, N. (2020). Cuando la falta de compromiso ocupacional del profesorado novel no es suficiente para explicar la intención de abandono: ¡la inteligencia emocional importa! Revista de Psicodidactica, 25(1), 52-58. https://doi.org/10.1016/j. psicod.2019.05.001

Míguez, M. (2010). Una estrategia didáctica alternativa en aulasuniversitarias de química: Potenciando el proceso motivacional por el aprendizaje. Educacion Quimica, 21(4), 278-286. https://doi.org/10.1016/s0187-893x(18)30096-x

Monti, A. (2020). De la planificación como técnica a la cultura de la planificación. EURE (Santiago), 46(137), 27-46. https://doi.org/http://dx.doi. org/10.4067/S0250-71612020000100027 
Morales Rodríguez, F. M. (2017). Relaciones entre afrontamiento del estrés cotidiano, autoconcepto, habilidades sociales e inteligencia emocional. European Journal of Education and Psychology, 10(2), 41-48. https://doi. org/10.1016/j.ejeps.2017.04.001

Moreno-Pinado, W. E., y Velázquez Tejeda, M. E. (2017). Estrategia Didáctica para Desarrollar el Pensamiento Crítico. REICE. Revista Iberoamericana Sobre Calidad, Eficacia $y$ Cambio En Educación, 15(2), 53-73. https://doi. org/10.15366/reice2017.15.2.003

Pallarés-Domínguez, D. (2016). Neuroeducación en diálogo: neuromitos en el proceso de enseñanza-aprendizaje y en la educación moral. Pensamiento, 72(23), 941-958. https://doi.org/ pen.v72.i273.y2016.010

Pasek, E., y Mejía, M. T. (2017). Proceso General para la Evaluación Formativa del Aprendizaje. Revista Iberoamericana de Evaluación Educativa, 10(1), 177-193. https://doi.org/https://doi. org/10.15366/riee2017.10.1.009

Piedra, I. D., Eraña, I. E., Segura-Azuara, N. de los Á., Hambleton, A., y López, M. V. (2018). Delineando criterios para la evaluación de tecnología educativa. Educacion Medica, 20(2), 108-113. https://doi.org/https://doi. org/10.1016/j.edumed.2018.04.020

Plos, T.D.(2019).Una enfermedad Neuroeducación : ¿real aporte al aprendizaje o mito ?, México. https://doi.org/doi.org/10.1371/ journal. pntd.0005004

Portellano Pérez, J. A. (2009). Cerebro Derecho, Cerebro Izquierdo. Implicaciones Neuropsicológicas delas AsimetríasHemisféricas en el Contexto Escolar. Psicologia Educativa, 15(1), 5-12. https://journals.copmadrid.org/ psed/art/9d2682367c3935defcb1f9e247a97c0d

Prieto, M. Á. (2011). Actividad física y salud. Innovacion $Y$ Experiencias Experiencias Educativas, (42), 11-42. https://archivos.csif.es/ archivos/andalucia/ensenanza/revistas/csicsif/ revista/pdf/Numero_42/MIGUEL_ANGEL_ PRIETO_BASCON_01.pdf

Romero, R., Cueva, H., y Barboza, L. (2014). La gimnasia cerebral como estrategia para el desarrollo de la creatividad en los estudiantes.
Omnia, 20(3), 80-91. http://www.redalyc.org/ articulo.oa?id=73737091006

Romero, J. (1996). El mito del hemisferio derecho del cerebro y la creatividad. Arte, Individuo y Sociedad, 1(8), 99-106. https://doi.org/10.5209/ ARIS.6841

Ruiz, J. (2018). La importancia de la formación docente en neuroeducación. Tesis de Grado, Universidad de Sevilla

Ruiz, L. V. (2016). Técnica del brain gym (gimnasia cerebral) para la motricidad fina y gruesa y su incidencia en el aprendizaje significativo en los y las estudiantes del inicial de la unidad educativa "dr. miguel h. alcívar" periodo 2016-2017. Universidad Técnica Estatal de Quevedo

Sánchez-López, D., León-Hernández, S. R., y Barragán-Velásquez, C. (2015). Correlación de inteligencia emocional con bienestar psicológico y rendimiento académico en alumnos de licenciatura. Investigación En Educación Médica, 4(15), 126-132. https://doi.org/10.1016/j. riem.2015.04.002

Sánchez, M. C., Gómez-Arteta, I. I., y Bonifaz, B. (2020). Predominio cerebral y rendimiento académico en los estudiantes de la facultad de educación de la Universidad Nacional del Altiplano (UNA)-Puno.Comuni@cción: Revista de Investigación En Comunicación y Desarrollo, 11(2), 97-106. https://doi.org/https://doi. org/10.33595/2226-1478.11.2.432

Seijo, C., y Barrios, L. (2012). El cerebro triuno y la inteligencia ética: matriz fundamental de la inteligencia multifocal. Praxis, 8(1), 147-165. https://doi.org/10.21676/23897856.40

Sindeev, A. (2018). Teoría del cerebro total: plena vigencia para el proceso de enseñanzaaprendizaje actual. Universidad Norbert Wiener, 7, 59-71.

Slimovich, A. (2021). La mediatización política durante la pandemia por COVID-19. La argumentación en las redes sociales de Alberto Fernández y Mauricio Macri. Dixit, 01(34), 01-14. https://doi.org/https://doi.org/10.22235/ d34.2273

Sosa, F. (2016). Actitud de los estudiantes, frente a la educación intercultural bilingüe - 2014. Revista de Investigaciones Altoandinas, 18(2), 231-236. 
https://doi.org/http://dx.doi.org/10.18271/ ria.2016.204

Sosa, F. (2018). Actitudes En La Formación Inicial Docente De La Región Puno. Revista de Investigaciones de La Escuela de Posgrado, 7(2), 580-591. https://doi.org/http://dx.doi. org/10.26788/riepg.2018.2.82

Sosa, F., y Vilca, H. M. (2021). Estrategias habituales de enseñanza en colegios rurales aimaras. Puriq, 3(2), 385-414. https://doi.org/https://doi. org/10.37073/puriq.3.2.174

Torres Toca, C. E. (2014). Inteligencia colectiva: enfoque para el análisis de redes. Estudios Gerenciales, 30(132), 259-266. https://doi. org/10.1016/j.estger.2014.01.014

Vargas Murillo, G. (2017). Recursos Educativos Didácticos En El Proceso Enseñanza Aprendizaje Educational Resources in the Process Teaching Learning. Revista "Cuadernos," 58(1), 68-74. http://www.revistasbolivianas.org.bo/pdf/chc/ v58n1/v58n1_a11.pdf

Velásquez B., B. M., Remolina De Cleves, N., y Calle M., M. G. (2005). Estrategias metodológicas facilitadoras del desarrollo del cerebro total y su incidencia en el aprendizaje significativo de los estudiantes universitarios. Tabula Rasa, 3(3), 315-338. http://www.redalyc.org/articulo. oa?id $=39600316$

Velásquez Burgos, B. M., Remolina de Cleves, N., y Calle Márquez, M. G. (2009). El cerebro que aprende. Tabula Rasa, 11, 329-347. http://www. redalyc.org/articulo.oa? $\mathrm{id}=39617332014$

Vergara, J. C., Maza, F., y Fontalvo, T. (2010). Futurología: origen, evolución y métodos. Revista Palobra, "Palabra Que Obra," 11(11), 218-229. https://doi.org/10.32997/2346-2884vol.11-num.11-2010-125

Vidarte, J. A., Vélez, C., Sandoval,C., y Alfonso, M. L. (2011). Hacia la Promoción de la salud. Hacia La Promoción de La Salud, 16(1), 202-218. https://doi.org/10.17151/hpsal.2019.24.2.1

Villada, M.A.,ySerna Guzmán, C. (2010).Innovando Estrategias metodológicas. En planeación para el desarrollo Revista. Revista Bitácora Urbano Territorial, 17(2), 145-160. http://www.redalyc. org/articulo.oa?id=74816991009

Vos, J. De. (2016). ¿Dónde está la educación en la neuroeducación? Teoría y Crítica de La Psicología, 8(1), 1-16. http://www.teocripsi. com/ojs/ 